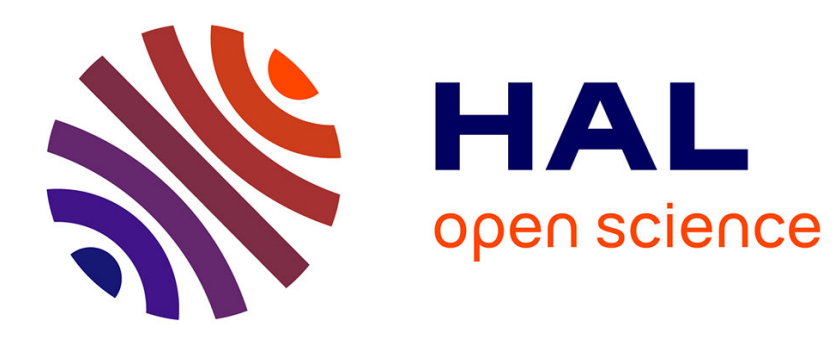

\title{
Approche économique de la conversion en région Centre
}

A. Triou, E. Vacherat

\section{To cite this version:}

A. Triou, E. Vacherat. Approche économique de la conversion en région Centre. Revue forestière française, 1989, 41 (5), pp.431-438. 10.4267/2042/25992 . hal-03425102

\section{HAL Id: hal-03425102 \\ https://hal.science/hal-03425102}

Submitted on 10 Nov 2021

HAL is a multi-disciplinary open access archive for the deposit and dissemination of scientific research documents, whether they are published or not. The documents may come from teaching and research institutions in France or abroad, or from public or private research centers.
L'archive ouverte pluridisciplinaire HAL, est destinée au dépôt et à la diffusion de documents scientifiques de niveau recherche, publiés ou non, émanant des établissements d'enseignement et de recherche français ou étrangers, des laboratoires publics ou privés. 


\title{
APPROCHE ÉCONOMIQUE DE LA CONVERSION EN RÉGION CENTRE
}

\author{
A. TRIOU - E. VACHERAT
}

Avant-propos

Après un siècle de discussions, est-il encore d'actualité de s'intéresser aux aspects économiques de la conversion?

En 1989, devant la tâche encore considérable qui reste à accomplir en matière de conversion, bien des communes et des particuliers hésitent à se lancer dans une telle aventure. Pourquoi? Les raisons à ce peu d'enthousiasme sont sans doute variables selon les catégories de propriétaires en cause, mais la raison essentielle n'est-elle pas qu'en matière forestière rien $n$ 'est définitivement acquis: les arguments en vogue au XIX $X^{\ominus}$ siècle, en faveur de la conversion, ont-ils la même valeur aujourd'hui?

Une actualisation de l'économie de la conversion est devenue nécessaire.

C'est la raison pour laquelle l'ENITEF a confié à Messieurs Triou et Vacherat, élèves de $3^{e}$ année, le soin de comparer les bilans matière (gros bois, bois moyens, petits bois et houppier-taillis) puis valeur (en francs 1985) des traitements en taillis-sous-futaie, en conversion et en futaie à partir de cas réels, c'est-à-dire par l'exploitation minutieuse des archives de plusieurs forêts domaniales et privées de la région Centre.

Ce travail de bénédictin des deux stagiaires a été rendu possible grâce à la collaboration efficace de l'Office national des Forêts - région Centre.

Nous laissons le soin au lecteur de l'article qui va suivre de découvrir les résultats quelque peu inattendus de cette étude, et nous sommes intéressés par toutes les réactions sur ce sujet ô combien essentiel pour la forêt française.

À l'heure où la polémique toujours latente au sujet du taillis-sous-futaie semble se ranimer, il nous a paru utile de faire état des principaux résultats de notre troisième année d'étude à I'ENITEF, année consacrèe à l'établissement de bilans " matière " et "argent " sur la conversion classique en futaie régulière de Chêne sessile dans la région Centre.

Rappelons que la conversion classique consiste à faire vieillir des peuplements de taillis-sousfutaie (TSF) dans le but d'accumuler du matériel de grande valeur d'une part, et d'autre part de constituer à terme une futaie régulière équilibrée par le jeu du renouvellement naturel. 
Dans une forêt en cours de conversion, trois traitements peuvent donc cohabiter : le taillis-sousfutaie, la conversion et la futaie régulière.

II nous a paru intéressant d'ètudier ces trois traitements séparément dans le cadre de séries distinctes équilibrées, puis globalement à l'échelle d'une seule série, au cours d'un cycle complet de conversion.

\section{TECHNIQUES ET MATÉRIAUX UTILISÉS}

Pour que les fondements de notre étude ne soient pas contestés, nous avons décidé de ne traiter que des cas réels pour lesquels les archives étaient suffisamment complètes pour nous permettre de reconstituer l'évolution des peuplements sur des durées significatives: révolution de 19 à 25 ans pour le taillis-sous-futaie, un siècle pour la conversion, 180 ans pour la futaie régulière. La validité des renseignements contenus dans les archives (il s'agissait principalement d'inventaires et de bilans d'exploitations) a été attestée par l'excellente concordance observée dans les résultats entre les différentes forêts étudiées.

Nous tenons à dire que notre travail a pu être mené à bien, dans de bonnes conditions, grâce à la qualité des archives qui nous ont été confiées et à la puissance du traitement informatique des données.

Le côté parfois fastidieux de nos travaux a ètė agrèmenté par l'émergence de résultats auxquels nous ne nous attendions pas tout à fait...

\section{DOMAINE ÉTUDIÉ}

Pour que les comparaisons entre les différents traitements soient valides, il nous a fallu choisir les parcelles ou groupes de parcelles (affectations) situées en conditions stationnelles équivalentes. Ces stations de type acidophile ou acidocline présentaient parfois des différences de fertilité attestées par des mesures de hauteur dominante, si bien que, pour plus de précision, nous avons distinguè pour les futaies régulières trois classes de fertilité ; la classe intermédiaire notėe F2 correspond à celle de la table de production de Pardé (1962).

En définitive, les parcelles que nous avons retenues pour notre étude et dont la surface totale avoisine le millier d'hectares, appartiennent aux forêts domaniales de Blois (Loir-et-Cher), Cîteaux (Loir-et-Cher), Loches (Indre-et-Loire) et Montargis (Loiret) pour la conversion et la futaie régulière, et à la forêt privée de Marchenoir (contiguë à la forêt de Cîteaux) pour le taillis-sousfutaie.

\section{CARACTÉRISTIQUES ÉTUDIÉES}

L'ètude du matériel ligneux exprimé globalement en mètre cube par hectare de bois fort, tel qu'il apparaît dans les tables de production, ne présente qu'un intérêt limité dans l'établissement de bilans économiques par mode de traitement:

- d'une part, parce que l'accroissement global est très peu influencé par le mode de traitement ;

- d'autre part parce qu'il est difficile de déterminer une valeur moyenne à attribuer à l'ensemble des catégories de produits aussi différentes que le taillis, le bois d'œuvre, les houppiers, etc... 
C'est la raison pour laquelle nous avons poussé nos investigations jusqu'au niveau " catégories de produits" telles qu'elles sont définies à l'Office national des Forêts pour l'estimation valeur des coupes.

Compte tenu des peuplements en cause où le Chêne domine très nettement, les six catégories retenues sont les suivantes:
1) Chêne : $\varnothing 15 \mathrm{~cm}$ et moins ;
4) Chêne : $\varnothing 50 \mathrm{~cm}$ et plus (GB);
2) Chêne : $\varnothing 20$ et $25 \mathrm{~cm}$;
5) Feuillus divers tous diamètres;
3) Chêne : $\varnothing 30$ à $45 \mathrm{~cm}(\mathrm{BM})$;
6) Houppier toutes essences et tous diamètres ;

Pour le taillis-sous-futaie, nous avons dû indiquer en outre la part de la charbonnette qui a été comptabilisée dans les coupes traditionnelles de taillis-sous-futaie mais que l'on néglige en futaie.

\section{ĖVOLUTION DES CATÉGORIES DE PRODUITS EN FUTAIE RÉGULIÈRE}

Les graphiques 1 et 2 (ci-contre) montrent l'évolution en volume des catègories de produits en fonction de l'âge des bois.

II est remarquable de constater que les gros bois $(\varnothing 50$ et + ) n'apparaissent qu'à 100 ou 120 ans selon la fertilité et que leur proportion s'accroît de façon considerable avec le temps.

\section{Graphiques 1 et 2}

ÉVOLUTION EN VOLUMES DES CATÉGORIES DE PRODUITS EN FONCTION DE L'ÁGE DES BOIS ET DE LA CLASSE DE FERTILITÉ.

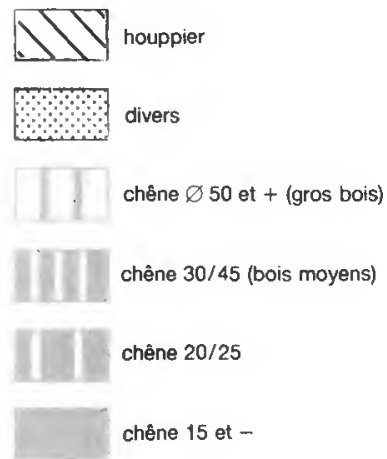

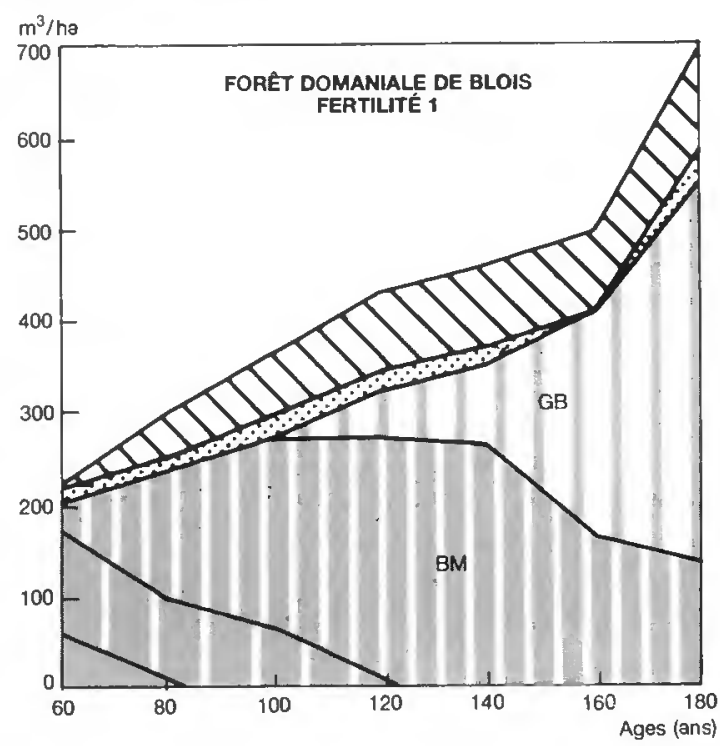

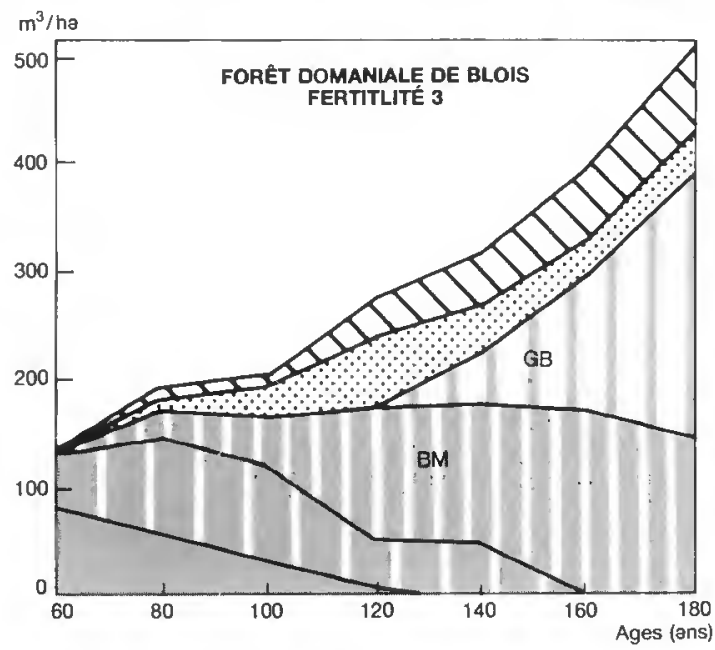




\section{A. TRIOU - E. VACHERAT}

Le graphique 3 (ci-contre) exprime l'évolution du volume des gros bois en fonction de l'âge de la futaie et de la classe de fertilité (notée F1, F2 ou F3). C'est au-delà de 140 ans que l'augmentation du volume à l'hectare des gros bois apparaît la plus rapide.

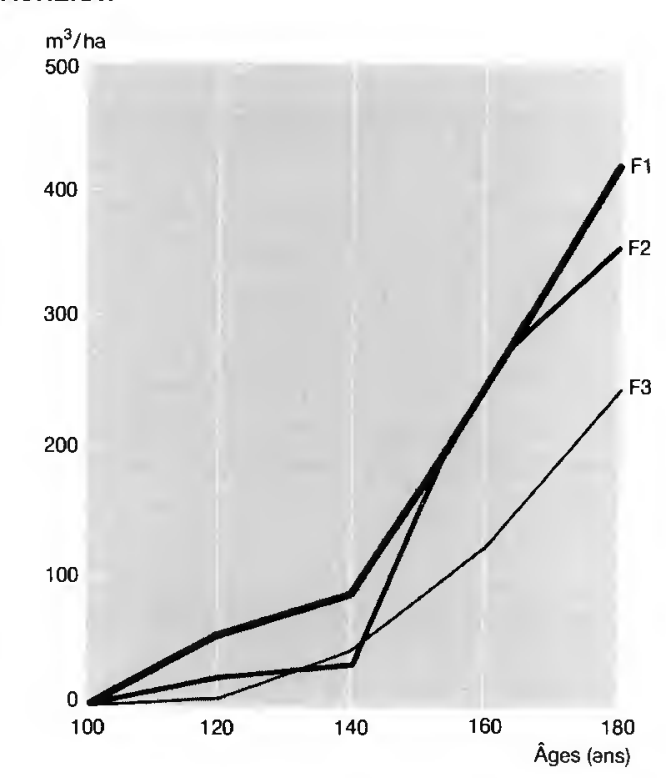

Graphique 3

ÉVOLUTION DU VOLUME DES GROS BOIS EN FONCTION DE L'ÂGE DE LA FUTAIE ET DE LA CLASSE DE FERTILITÉ.

On imagine donc aisément que le revenu "argent "qui dépend dans une large mesure de la quantité de gros bois produite, sera une fonction directe de l'âge d'exploitabilité de la futaie régulière, et que la tendance actuelle qui consiste à réduire le terme d'exploitabilité des chênaies risque d'être une erreur économique.

Nous nous sommes bien évidemment préoccupés de savoir si une sylviculture plus dynamique pouvait laisser espérer une réduction de l'âge d'exploitabilité pour le Chêne sessile. Les conclusions de la Recherche française et étrangère ne sont pas optimistes: les arbres dominants, producteurs quasi exclusifs de gros bois ont une croissance en diamètre qui n'est que peu influencée par l'intensité des éclaircies. II nous a d'ailleurs été donné de vérifier concrètement cette importante conclusion de la Recherche lors de comparaison de parcelles ayant subi des éclaircies de nature et d'intensité très différentes, car ni les éclaircies fortes et précoces ni la désignation de tiges d'avenir ne sont des inventions récentes.

\section{Graphique 4}

\section{ÉVOLUTION DES CATÉGORIES DE PRODUITS EN CONVERSION.}

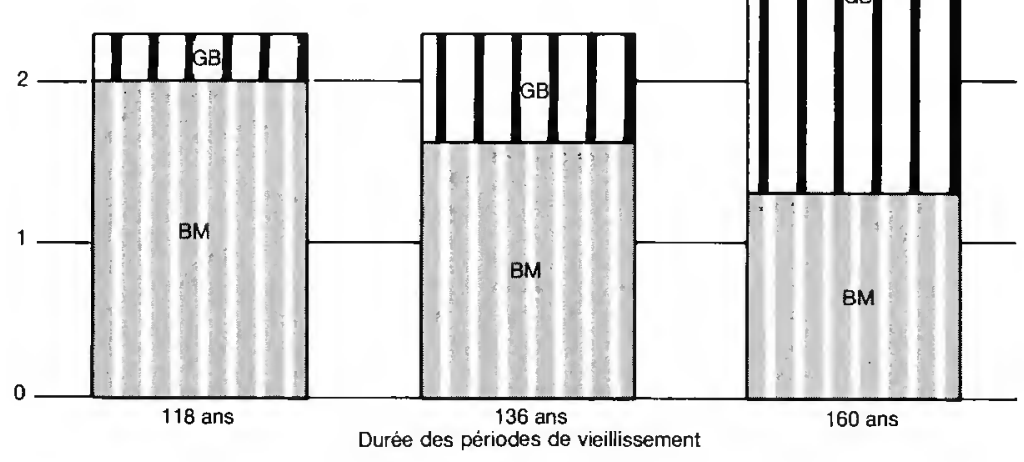




\section{ÉVOLUTION DES CATÉGORIES DE PRODUITS EN CONVERSION}

Lors de la période du vieillissement du taillis-sous-futaie en vue de l'obtention d'une futaie sur souche (FSS), l'évolution des catégories de produits est tout à fait comparable à celle observée en futaie règulière $(F)$. Le graphique 4 (p. 434, en bas) montre la production moyenne annuelle de bois moyens (BM, $\varnothing 30$ à 45$)$ et de gros bois (GB, $\varnothing 50$ et + ) après des périodes de vieillissement de 118 ans, 136 ans et 160 ans.

L'augmentation rapide des gros bois est là aussi surtout sensible après 140 ans.

\section{BILANS “ MATIÈRE "}

Le tableau I présente les productions moyennes annuelles par hectare réellement constatées en taillis-sous-futaie (TSF), en futaie régulière vraie (F) à 120 ans et 180 ans, et en futaie sur souche (FSS) issue de vieillissement d'un taillis-sous-futaie pendant 118,136 et 160 ans.

Les rèsultats sont exprimès en $\mathrm{m}^{3} / \mathrm{ha} / \mathrm{an}$.

Tableau | Production (en $\mathrm{m}^{3} / \mathrm{ha} / \mathrm{an}$ ) des traitements par catégories en fonction de l'âge

\begin{tabular}{|c|c|c|c|c|c|c|}
\hline Catégories & $\begin{array}{c}\text { Taillis- } \\
\text { sous-futaie }\end{array}$ & $\begin{array}{l}\text { Futaie } \\
120 \text { ans }\end{array}$ & $\begin{array}{l}\text { Futaie } \\
180 \text { ans }\end{array}$ & $\begin{array}{c}\text { Futaie } \\
\text { sur souche } \\
118 \text { ans }\end{array}$ & $\begin{array}{c}\text { Futaie } \\
\text { sur souche } \\
136 \text { ans }\end{array}$ & $\begin{array}{c}\text { Futaie } \\
\text { Sur souche } \\
160 \text { ans }\end{array}$ \\
\hline Chêne 50 et $+\ldots \ldots \ldots \ldots \ldots \ldots$ & 0,9 & 0,1 & 2,0 & 0,3 & 0,7 & 2,3 \\
\hline Chêne $30 / 45 \ldots \ldots \ldots \ldots \ldots \ldots$ & 0,4 & 1,9 & 1,4 & 2,0 & 1,6 & 1,3 \\
\hline Chêne 15 et $-\ldots \ldots \ldots \ldots \ldots \ldots$ & \multirow{4}{*}{2,7} & 0,4 & 0,3 & \multirow{2}{*}{0,6} & \multirow{2}{*}{0,5} & \multirow{2}{*}{0,5} \\
\hline Chêne $20 / 25 \ldots \ldots \ldots \ldots \ldots$ & & 0,9 & 0,5 & & & \\
\hline Divers $\ldots \ldots \ldots \ldots \ldots \ldots \ldots \ldots$ & & 0,2 & 0,2 & 0,4 & 0,4 & 0,3 \\
\hline Houppier ................... & & 0,5 & 0,7 & 0,7 & 0,7 & 0,9 \\
\hline Charbonnette $\ldots \ldots \ldots \ldots \ldots \ldots$ & 1,3 & $\varepsilon$ & $\varepsilon$ & $\varepsilon$ & $\varepsilon$ & $\varepsilon$ \\
\hline Total $\ldots \ldots \ldots \ldots \ldots \ldots$ & 5,3 & 4,0 & 5,1 & 4,0 & 3,9 & 5,3 \\
\hline
\end{tabular}

Ce tableau appelle les remarques suivantes:

- La production totale semble s'établir entre 5,1 et $5,3 \mathrm{~m}^{3} / \mathrm{ha} / \mathrm{an}$, quel que soit le traitement. Les productions inférieures constatées en F 120 ans, FSS 118 ans et FSS 136 ans s'expliquent par le fait qu'à ces âges peu avancés la proportion de charbonnette produite, qui n'est pas encore négligeable, n'a pas été comptabilisée dans les coupes contrairement à ce qui se passait à l'époque en taillis-sous-futaie.

- À 120 ans en futaie et à 118 ans en futaie sur souche, la production de gros bois est dérisoire ; à 136 ans elle est encore inférieure à celle du taillis-sous-futaie!

- La futaie à 180 ans et la futaie sur souche à 160 ans ont produit respectivement 3,4 et $3,6 \mathrm{~m}^{3} /$ ha/an de bois d'œuvre $(\varnothing 30 \mathrm{~cm}$ et + ), soit trois fois plus que le taillis-sous-futaie, mais seulement 2,5 fois plus si l'on ne tient compte que des seuls gros bois (50 et + ).

- Les différences constatées sur la production de gros bois entre la futaie réguliere à 180 ans et la futaie sur souche à 160 ans, en faveur de cette dernière, sont dues, nous l'avons vérifié, à la purge de gros bois présents dans le taillis-sous-futaie au début de la phase de vieillissement. 
II faut remarquer, et c'est là un point qui nous paraît important, que les productions sont sensiblement les mêmes en futaie sur souche qu'en futaie vraie : il semble donc que le peu de matériel initial fourni à l'origine par le taillis-sous-futaie puisse rentabiliser le sol autant qu'une régénération naturelle ou artificielle! La technique de la conversion par balivage intensif, technique ancienne qui jouit d'un regain d'intérêt actuellement, semble donc tout à fait fondèe.

\section{BILANS ÉCONOMIQUES}

Les bilans que nous avons établis font apparaitre les revenus annuels moyens par hectare, bruts et nets, obtenus sur des séries équilibrées, dans le contexte économique de 1985. Les revenus bruts ont été obtenus par simple application de prix unitaires à chaque catégorie de produits. Les revenus nets ont été plus délicats à appréhender en raison des hypothèses que nous avons dû faire au sujet des charges (gestion, imposition, travaux...). Pour plus de précision sur l'estimation de nos prix unitaires et sur nos hypothèses de coûts de gestion, nous incitons le lecteur à consulter notre mémoire.

Le graphique 5 ( $p .437$ ) permet la comparaison des revenus bruts (exprimés en francs 1985 par hectare et par an) fournis par les différentes catégories de produits ligneux, entre le taillis-sousfutaie et la futaie régulière à 120 et 180 ans. À 120 ans, la futaie régulière procure un revenu brut équivalent à celui du taillis-sous-futaie, mais à 180 ans ce revenu est trois fois supérieur.

Les revenus nets, dans l'hypothèse d'un coût de gestion évaluè à $10 \%$ des revenus bruts, s'établissent à $582 \mathrm{~F} / \mathrm{ha} /$ an pour le taillis-sous-futaie et à $1438 \mathrm{~F} / \mathrm{ha} / \mathrm{an}$ pour la futaie régulière à 180 ans, soit un rapport de 1 à 2,5 .

Dans le calcul des revenus nets, nous n'avons pas tenu compte des frais de succession, très élevés en futaie régulière, et donc d'importance capitale en forêt privée.

\section{LE SCÉNARIO DE LA CONVERSION CLASSIQUE}

À partir de deux cas réels (forêts de Blois et Cîteaux), nous avons construit un modèle complet d'évolution d'une série de conversion depuis le taillis-sous-futaie jusqu'à l'obtention d'une futaie régulière équilibrẻe. Le graphique 6 (p. 437) montre l'évolution des récoltes exprimées en $\mathrm{m}^{3} / \mathrm{ha} /$ an, toutes catégories de produits confondues sur l'ensemble du cycle. La charbonnette n'a pas été comptabilisèe, ce qui explique la différence d'environ un $\mathrm{m}^{3} / \mathrm{ha} /$ an entre taillis-sous-futaie et futaie.

Ce cycle de 305 ans se compose de 4 époques:

- Époque $1: 0$ - 125 ans

Cette époque correspond à l'accumulation du matériel ligneux rẻalisẻe au cours des phases de vieillissement. La diminution progressive de la récolte observèe pendant cette époque est due aux entrées successives des affectations en phase de vieillissement.

\section{- Époque 2: 125 - 275 ans}

Celle-ci présente deux caractéristiques:

- la récolte importante est due aux coupes de conversion. Ces coupes réalisent le matériel ligneux accumulé depuis 125 ans ;

- la récolte augmente régulièrement car les éclaircies de jeune futaie s'ajoutent progressivement aux coupes de conversion. 


\section{Graphique 5 \\ COMPARAISON DES \\ REVENUS BRUTS \\ EN TAILLIS-SOUS-FUTAIE \\ ET EN FUTAIE.}$$
\text { F/ha/an }
$$$$
2000
$$

1500

1000

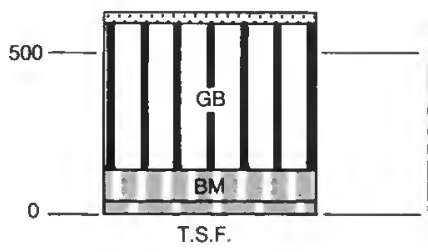

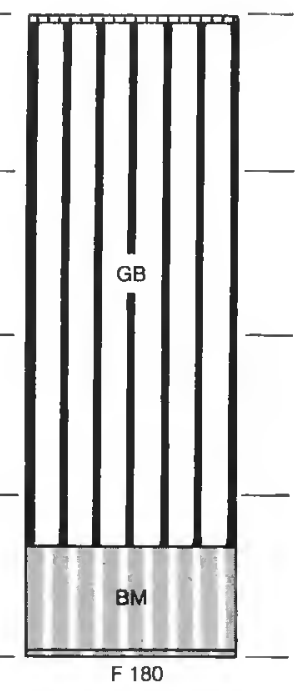

\section{- Époque 3 : 275 - 305 ans}

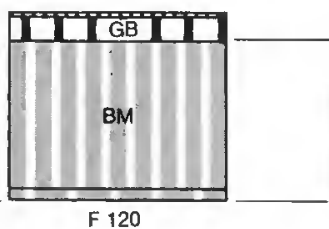

Cette époque est appelée trou de production. Elle se caractérise par l'absence de coupes de régénération. Les coupes de conversion sont terminées et les premières coupes de régénération de futaie ne sont pas commencées car l'âge d'exploitabilité est fixé à 180 ans. La récolte faible et peu rémunératrice est uniquement le fait des coupes d'amélioration de futale.

\section{- Époque 4 : 305 ans et au-delà}

C'est la futaie équilibrée: la récolte est régulière et égale à la production.

On s'aperçoit que :

- la durée de la conversion est très longue: trois siècles! II est vrai que la conversion directe permettrait de réduire cette durée de moitié mais au prix d'une longue période déficitaire :

- les revenus à espérer au cours de cette durée sont très Irréguliers, ce qui peut être une gêne considerable pour les forêts communales et privées.

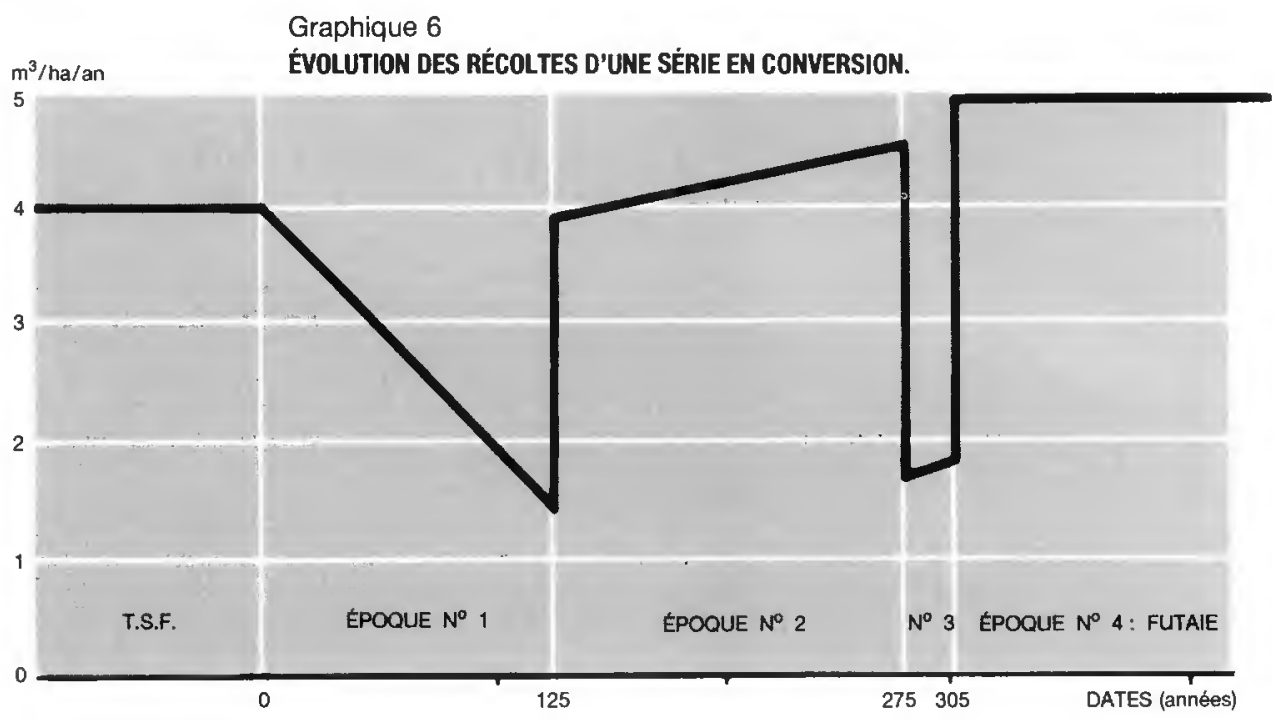




\section{A. TRIOU - E. VACHERAT}

\section{CONCLUSIONS}

Nos conclusions ne concernent bien évidemment que les forêts de Chêne de la région Centre.

- La futaie régulière n'est économiquement intéressante qu'à long terme. L'expérience montre que ni les éclaircies fortes, ni la désignation ne permettent d'envisager une réduction notable de l'âge d'exploitabilité.

- La conversion, qu'elle soit classique ou directe, s'accompagne d'une longue pèriode de "vaches maigres" pendant laquelle les revenus sont inférieurs à ceux du taillis-sous-futaie. ॥ faut en effet pendant cette période tripler le volume du capital producteur.

Pour les forêts privées, les droits de succession, qui sont fonction de la valeur des bois sur pied, vont pénaliser très lourdement le régime de la futaie.

- La production des taillis-sous-futaie que nous avons étudiés nous a paru appréciable eu égard à la gestion extensive à laquelle ils ont èté soumis. En effet, sur les quelque 600 ha de peuplements que nous avons étudiés, le volume grume après la coupe n'excédait pas $30 \mathrm{~m}^{3} / \mathrm{ha}$. Pourtant la production constatee en gros bois $(\varnothing 50$ et +$)$ pour des révolutions moyennes de 24 ans a été de $0,9 \mathrm{~m}^{3} / \mathrm{ha} / \mathrm{an}$, chiffre qu'il faut comparer aux $2 \mathrm{~m}^{3} / \mathrm{ha} / \mathrm{an}$ produits par la futaie à 180 ans. La valeur des produits d'une futaie est certes supérieure à celle des produits d'un taillis-sous-futaie mais globalement les prestations de la futaie régulière nous ont quelque peu déçus.

\begin{tabular}{|c|}
\hline A. TRIOU \\
OFFICE NATIONAL DES FORÊTS \\
Cité administrative \\
Esplanade du Mail \\
81100 CASTRES \\
\hline DIRECTION DÉPARTEMENTALE DE L'AGRICULTURE \\
ET DE LA FORÉT DE L'AUBE \\
Caserne de Beurnonville \\
Rue de Turenne \\
10026 TROYES CÉDEX
\end{tabular}

